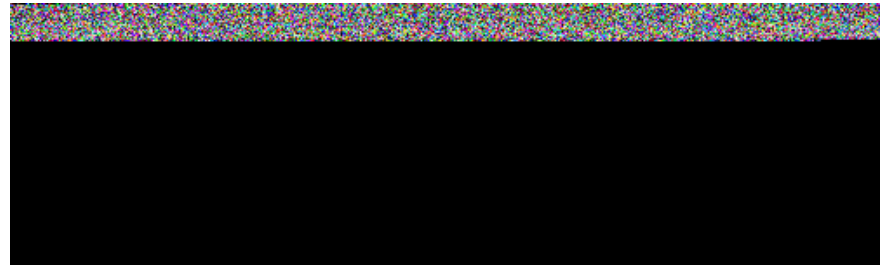

This information is current as of April 26, 2023.

\title{
A Radiologic Score to Distinguish Autoimmune Hypophysitis from Nonsecreting Pituitary Adenoma Preoperatively
}

\author{
A. Gutenberg, J. Larsen, I. Lupi, V. Rohde and P. Caturegli \\ AJNR Am J Neuroradiol 2009, 30 (9) 1766-1772 \\ doi: https://doi.org/10.3174/ajnr.A1714 \\ http://www.ajnr.org/content/30/9/1766
}




\section{ORIGINAL RESEARCH}

A. Gutenberg

J. Larsen

I. Lupi

V. Rohde

P. Caturegli

\title{
A Radiologic Score to Distinguish Autoimmune Hypophysitis from Nonsecreting Pituitary Adenoma Preoperatively
}

\begin{abstract}
BACKGROUND AND PURPOSE: Autoimmune hypophysitis (AH) mimics the more common nonsecreting pituitary adenomas and can be diagnosed with certainty only histologically. Approximately $40 \%$ of patients with $\mathrm{AH}$ are still misdiagnosed as having pituitary macroadenoma and undergo unnecessary surgery. MR imaging is currently the best noninvasive diagnostic tool to differentiate $\mathrm{AH}$ from nonsecreting adenomas, though no single radiologic sign is diagnostically accurate. The purpose of this study was to develop a scoring system that summarizes numerous MR imaging signs to increase the probability of diagnosing $\mathrm{AH}$ before surgery.
\end{abstract}

\begin{abstract}
MATERIALS AND METHODS: This was a case-control study of 402 patients, which compared the presurgical pituitary MR imaging features of patients with nonsecreting pituitary adenoma and controls with $\mathrm{AH}$. MR images were compared on the basis of 16 morphologic features besides sex, age, and relation to pregnancy.
\end{abstract}

RESULTS: Only 2 of the 19 proposed features tested lacked prognostic value. When the other 17 predictors were analyzed jointly in a multiple logistic regression model, 8 (relation to pregnancy, pituitary mass volume and symmetry, signal intensity and signal intensity homogeneity after gadolinium administration, posterior pituitary bright spot presence, stalk size, and mucosal swelling) remained significant predictors of a correct classification. The diagnostic score had a global performance of 0.9917 and correctly classified $97 \%$ of the patients, with a sensitivity of $92 \%$, a specificity of $99 \%$, a positive predictive value of $97 \%$, and a negative predictive value of $97 \%$ for the diagnosis of $\mathrm{AH}$.

CONCLUSIONS: This new radiologic score could be integrated into the management of patients with $\mathrm{AH}$, who derive greater benefit from medical as opposed to surgical treatment.

A denomas of the pituitary gland are the most common intracranial neoplasm, with a population prevalence of $0.1 \%{ }^{1}$ and autopsy prevalence of $15 \% .^{2}$ Approximately $65 \%$ of pituitary adenomas secrete a hormone ( $48 \%$ prolactin, $10 \%$ growth hormone, $6 \%$ corticotropin, and 1\% thyrotropin) causing typical hypersecretory syndromes. ${ }^{3}$ The remaining $(35 \%)$ pituitary adenomas do not produce (or secrete) a hormone and are thus referred to as nonfunctioning (or nonsecreting) adenomas.

Nonsecreting pituitary adenomas are typically macroadenomas (diameter, $>10 \mathrm{~mm}$ ) and lack clinical or biochemical evidence of hormonal excess. They derive most commonly from the gonadotrophs, ${ }^{4}$ though each pituitary cell type can give rise to tumors that are clinically silent. ${ }^{5}$ Nonsecreting adenomas present with neurologic symptoms due to the mass effect on structures surrounding them, such as visual distur-

\section{Received November 21, 2008; accepted after revision April 27, 2009}

From the Departments of Neurosurgery (A.G., V.R.) and Neuroradiology (J.L.), Georg August University Göttingen, Göttingen, Germany; Department of Endocrinology and Metabolism (I.L.), University of Pisa, Pisa, Italy; and Department of Pathology (P.C.), the Johns Hopkins University, Baltimore, Md.

This work was supported in part by the National Institutes of Health grants DK55670 and DK080351 to P.C. A.G. was supported by the Research Program from the Faculty of Medicine, Georg-August-University Göttingen, Göttingen, Germany, and the Tönnis-Stipendium by the German Society for Neurosurgery.

Please address correspondence to Angelika Gutenberg, MD, Department of Neurosurgery, Georg August University, Robert-Koch-Str 40, D-37099 Göttingen, Germany; e:mail: agutenberg@med.uni-goettingen.de

Indicates open access to non-subscribers at www.ajnr.org

Indicates article with supplemental on-line table

DOI 10.3174/ajnr.A1714 bances, headache ${ }^{6}$ or pituitary deficiencies ${ }^{7}$ or as incidental masses discovered on radiologic studies performed for other reasons. In this context, it is, however, important to realize that there are other nonadenomatous nonsecreting masses of pituitary origin for which surgery is not always indicated. Hypophysitis is an emerging disease to consider in this category.

Hypophysitis comprises 2 main histopathologic forms: lymphocytic and granulomatous. ${ }^{8}$ Lymphocytic hypophysitis, the most commonly encountered form, has a well-established autoimmune pathogenesis, predominantly affects women, and frequently presents during late pregnancy or in the early postpartum period. ${ }^{9}$ Granulomatous hypophysitis has different epidemiologic features, including lack of both female bias and association with pregnancy and a more aggressive clinical course. Its pathogenesis remains uncertain, though $\mathrm{McKeel}^{10}$ considered the 2 forms as different stages of the same disease. Lymphocytic and granulomatous hypophysitis, which will be collectively referred to as autoimmune hypophysitis $(\mathrm{AH})$ for the purpose of this article, both induce clinical and radiologic abnormalities that resemble those of nonsecreting pituitary adenomas very closely.

Although the autoimmune nature of $\mathrm{AH}$ is well established, the pathogenic autoantigens targeted in this disease remain to be identified. A reliable serologic test based on implicated autoantibodies is, thus, not yet available. ${ }^{11}$ Consequently, a diagnosis of AH can only be achieved with certainty by histologic examination of the pituitary gland, which necessitates an invasive approach. At present, patients with $\mathrm{AH}$ frequently undergo surgery for a presumptive diagnosis of pituitary adenoma. ${ }^{12}$ Differentiating $\mathrm{AH}$ from nonsecreting pituitary adenomas before surgery would, therefore, greatly 
benefit affected individuals because $\mathrm{AH}$ can often be successfully treated with lympholytic medications alone, ${ }^{13}$ whereas adenomas do indeed usually require surgical resection, ${ }^{14}$ conventionally via the trans-sphenoidal route. ${ }^{15}$

MR imaging is the procedure of choice in the evaluation of sellar masses, ${ }^{16}$ and sequencing recommendations comprise pre- and postgadolinium enhanced thin-section $(<3 \mathrm{~mm})$ sagittal and coronal T1-weighted images with optional T2weighted or fat-suppressed sequences. ${ }^{17} \mathrm{MR}$ imaging features more indicative of $\mathrm{AH}$ include a symmetric enlargement of the pituitary gland, a thickened nontapering pituitary stalk, and an intact sellar floor. ${ }^{18,19}$ In contrast, pituitary macroadenomas are frequently asymmetric, often displacing the infundibulum, and rarely involve the stalk or erode the sellar floor. ${ }^{20}$ In addition, macroadenomas appear heterogeneous both before and after contrast medium administration, in direct relationship to their size, though heterogeneity can also occur in $\mathrm{AH}^{21-23}$

Overall, no single radiologic sign has sufficient accuracy to distinguish with certainty $\mathrm{AH}$ from pituitary adenomas. The aim of this study, therefore, was to develop a diagnostic scoring system from a wide range of clinical and MR imaging features to increase the probability of diagnosing $\mathrm{AH}$ before surgery.

\section{Materials and Methods}

\section{Study Design and Patients}

This was a case-control study of 402 patients, which compared the presurgical pituitary MR imaging features of patients with nonsecreting pituitary adenoma and controls with $\mathrm{AH}$.

Patients with nonsecreting pituitary adenomas $(n=98)$ consecutively underwent surgery at the Johns Hopkins hospital and were selected from the surgical pathology data base on the basis of a histopathologic diagnosis of prolactin-, growth hormone-, corticotropin-, and thyrotropin-negative macroadenoma and the absence of elevated anterior pituitary hormones (except for mild hyperprolactinemia). There were no patients with histologic signs of hemorrhagic infarction.

Patients with AH $(n=304)$ were selected from the Johns Hopkins hospital surgical pathology archive ( $n=3$, all lymphocytic) and from the published literature ( $n=301$ ) by using 3 sources: 1 ) PubMed and Google Scholar searches by using the following keywords: "hypophysitis," "adenohypophysitis," "infundibul*," "autoimmu* and hypophys*," "lympho* and hypophys*," "granulom* and hypophys, "autoimmu* and pituit ${ }^{*}$, " "MR imaging and pituit*," and "MR imaging, and sella"; 2) citations in the articles identified above; and 3) citations in numerous textbook chapters.

For the published patients with primary lymphocytic hypophysitis, we identified 530 articles published from January 1962 to March 2008. The articles were written in English $(n=426)$, Japanese $(n=$ $50)$, French $(n=18)$, Spanish $(n=10)$, Korean $(n=7)$, German $(n=6)$, Chinese $(n=3)$, Portuguese $(n=3)$, Italian $(n=2)$, Dutch $(n=2)$, Polish $(n=1)$, and Czech $(n=1)$. The articles included 291 single case reports and 79 small case series, for a total of 471 patients. Of them, 255 patients had MR imaging descriptions sufficiently detailed to be included in the study. The diagnosis was established by surgical pathology in 152 patients and on clinical and radiologic grounds in 103 patients.

In the Johns Hopkins hospital surgical pathology archive, 26 hy- pophysitis cases were identified among 1459 pituitary specimens examined between January 1988 and March 2008 (23 purely lymphocytic and 3 with mixed granulomatous and lymphocytic features). Of these 26 cases, 11 underwent surgery at the Johns Hopkins hospital, and 15 were operated elsewhere but had their pituitary slides sent to Johns Hopkins for review. A presurgical MR imaging study was available in 3 of the 11 cases, all lymphocytic.

For the published patients with primary granulomatous hypophysitis, we identified 71 articles in English from 1969 to March 2008, describing a total of 66 patients. Of these, 46 cases, all histologically proved, had a sufficiently detailed MR imaging description to be included in the study.

Three clinical features were recorded for each patient: age, sex, and relation to pregnancy at the time of the initial symptoms (on-line Table). The relation to pregnancy was coded as 1 when present; 0 when absent in women of reproductive age; and not applicable in preteen girls, women older than 50 years of age, and males.

\section{Pituitary MR Imaging Features}

A total of 16 pituitary MR imaging features were evaluated in each patient (on-line Table). Patients with $\leq 4 \mathrm{MR}$ imaging features or no MR imaging evidence of a pituitary mass (pituitary volume, $<1 \mathrm{~cm}^{3}$ ) were excluded from the study. If a particular feature was not reported in the original publication, it was coded as missing. The volume of the pituitary mass (in cubic centimeters) was calculated by multiplying lesion height, width, and length. Lesion volume and patient age were the only continuous covariates in this study. Lesion volume was then dichotomized, assigning a value of 0 for volumes $<7 \mathrm{~cm}^{3}$ and 1 for volumes $\geq 7 \mathrm{~cm}^{3}$. T1 signal intensity (ie, the recovery of longitudinal magnetization) was classified as isointense, hypointense (which included isohypointense), or hyperintense (including isohyperintense) in relation to the intensity of gray matter on precontrast images. ${ }^{21,24}$ Homogeneity (ie, the absence of a focus of signal-intensity alteration separable from the remaining normal tissue $)^{25}$ was classified as homogeneous, heterogeneous (including cystic), and centrally hypointense (including ring enhancement). The intensity of gadolinium enhancement was classified into low or high compared with the anterior pituitary and/or cavernous sinuses. The features of gadolinium enhancement were classified as homogeneous, heterogeneous, and central hypoattenuated.

The symmetry (ie, the configuration of the pituitary gland on coronal sections) was classified as asymmetric (side-to-side shift) or symmetric. ${ }^{25}$ The posterior pituitary bright signal intensity (ie, the normal hyperintensity of the posterior pituitary) was classified as conserved or lost. ${ }^{25}$ The pituitary stalk was described as normal, thickened, or not identifiable. A normal pituitary stalk has a transverse diameter of $3.25 \pm 0.56 \mathrm{~mm}$ at the level of the optic chiasm and measures $1.91 \pm 0.4 \mathrm{~mm}$ at the pituitary insertion. ${ }^{26}$ The enhancement of the pituitary stalk after gadolinium administration was scored in relation to the neurohypophysis or the optic chiasm and classified into normal isointense (the stalk does not normally enhance) or abnormal hyperintense. ${ }^{26}$ Hypothalamic involvement was described as physiologically isointense or having a pathologically high gadolinium enhancement. ${ }^{27}$

Finally, 5 features of the parasellar region were considered as present or absent: dural tail (a tapering rim of enhancing dura mater extending from the mass ${ }^{28}$ ), thickening of the mucosa lining the sphenoid and adjacent posterior ethmoid sinuses (given that the mucosa of normally ventilated paranasal sinuses is indistinguishable on preor postcontrast MR images, any swelling or enhancement was consid- 
ered pathologic), erosion of the sellar floor (bone does not typically return signal intensity on MR imaging, so this feature was considered only when CT was also performed), invasion of the cavernous sinus, involvement of the visual pathways (defined by swelling or displacement of the optical nerves or chiasm), and involvement of the cavernous part of the internal carotid artery (only considered when either flow voids were described or angiography was performed).

\section{Statistical Analysis}

The study included 1 dichotomous outcome, coded 1 for a diagnosis of nonsecreting pituitary macroadenoma and 0 for hypophysitis, and analyzed how this diagnosis could be predicted by a set of 3 clinical (sex, age, and relation to pregnancy) and 16 radiologic covariates (on-line Table).

The contribution of each individual covariate was evaluated by univariate logistic regression. Next, missing values present in the various covariates (on-line Table) were derived by multiple imputations by using the imputation by chained equations approach. ${ }^{29}$ Finally, the covariates were evaluated by multivariate logistic regression to choose a model that best predicted the correct diagnosis. Selection of the significant covariates was performed by using the backward procedure. $^{30}$ The regression coefficient of each significant covariate was used to create a score by assigning to each covariate a signed number proportional to its regression coefficient. A positive number suggested a diagnosis of adenoma (which was coded as 1), whereas a negative number, a diagnosis of hypophysitis (coded as 0 ). Covariate scores were then added to calculate a cumulative score for each patient.

The accuracy of the score in classifying correctly the outcome as adenoma or hypophysitis was evaluated by using the receiver operating characteristic (ROC) analysis. This analysis computes the sensitivity and specificity of a diagnostic test (in this case the score) by using each value of the rating as a possible classification cut-point. The resulting sensitivity and 1-specificity values are then plotted on a graph and joined by straight lines to form the ROC curve. The area under the curve (AUC) is finally calculated by using the trapezoidal rule to summarize the global performance of the diagnostic test. An AUC of 1.0 would indicate a perfect score (in this case, a score that always classifies patients correctly as having adenoma or hypophysitis), whereas an AUC of 0.5 would classify patients at random.

All statistical analyses were performed by using STATA software, Release 10.0 (StataCorp, College Station, Tex).

\section{Results}

When analyzed individually, 17 of the 19 clinical and MR imaging features were significantly associated with the outcome (on-line Table). The 2 features that lacked diagnostic value were the dural tail sign and hypothalamic involvement (online Table).

When analyzed collectively in a multiple logistic regression model, 8 features contributed significantly to classifying the outcome as pituitary adenoma or hypophysitis: relation to pregnancy, pituitary mass volume and symmetry, signal intensity and signal-intensity homogeneity after gadolinium administration, posterior pituitary bright spot presence, stalk size, and mucosal swelling (Table). Figure 1 illustrates 1 case of $\mathrm{AH}$ with these radiologic features. These 8 predictors were used to build the final multiple logistic regression model that yielded the $\beta$ coefficients and odds ratios reported in the Ta-

\begin{tabular}{|c|c|c|c|c|c|}
\hline \multicolumn{6}{|c|}{$\begin{array}{l}\text { Table: Multiple logistic regression model based on } 9 \text { covariates } \\
\text { used to predict before surgery pituitary adenoma (coded as } 1 \text { ) or } \\
\text { hypophysitis (coded as } 0 \text { ) }\end{array}$} \\
\hline & $\beta^{*}$ & OR & $95 \% \mathrm{Cl}$ & $P$ & Score \\
\hline \multicolumn{6}{|l|}{ Age (yr) } \\
\hline$\leq 30$ & -1.70 & 0.18 & $0.029-1.12$ & .067 & -1 \\
\hline \multicolumn{6}{|c|}{ Relation to pregnancy } \\
\hline Yes & -3.71 & 0.03 & $0.002-0.42$ & .009 & -4 \\
\hline \multicolumn{6}{|c|}{ Pituitary volume $\left(\mathrm{cm}^{3}\right)$} \\
\hline$\geq 6$ & 1.83 & 5.12 & $1.98-16.28$ & .003 & 2 \\
\hline \multicolumn{6}{|c|}{ Gd enhancement type } \\
\hline Medium or high & -1.32 & 0.59 & $0.07-0.65$ & .011 & -1 \\
\hline \multicolumn{6}{|c|}{ Gd enhancement features } \\
\hline Heterogeneous & 1.41 & 4.31 & $1.34-35.8$ & .041 & 1 \\
\hline \multicolumn{6}{|l|}{ Symmetry } \\
\hline Asymmetric & 2.49 & 12.1 & $2.92-49.7$ & .001 & 3 \\
\hline \multicolumn{6}{|c|}{ Posterior pituitary bright spot } \\
\hline Lost & -2.42 & 0.09 & $0.015-0.51$ & .007 & -2 \\
\hline \multicolumn{6}{|l|}{ Stalk size } \\
\hline Enlarged & -5.34 & 0.005 & $0.0004-0.06$ & $<.001$ & -5 \\
\hline \multicolumn{6}{|l|}{ Mucosal thickening } \\
\hline Present & 2.15 & 8.61 & $1.25-58.9$ & .028 & 2 \\
\hline
\end{tabular}

Note:- $0 \mathrm{R}$ indicates odds ratio; $\mathrm{Cl}$, confidence interval.

${ }^{*}$ Regression coefficient. A signed score was assigned to each covariate proportional to its regression coefficient.

ble. Age, although only borderline significant, was retained in the final model because of its clinical relevance.

Age tended to be greater in patients with nonsecreting pituitary adenoma than in those with $\mathrm{AH}$, with peak frequency distributions at 60 and 32 years, respectively (Fig $2 A$ ). In particular, ages younger than 30 years significantly predicted a diagnosis of hypophysitis rather than adenoma (odds ratio, $0.18 ; P=.067$; Table).

Appearance of symptoms during late pregnancy or the early postpartum period strongly favored a diagnosis of $\mathrm{AH}$ rather than adenoma (odds ratio, 0.03; $P=.009$; Table).

Pituitary volume was significantly greater in adenoma than in $\mathrm{AH}$, with median values of $10 \mathrm{~cm}^{3}$ and $3 \mathrm{~cm}^{3}$, respectively (Fig $2 B$ ). Volumes $>6 \mathrm{~cm}^{3}$ significantly predicted a diagnosis of adenoma (odds ratio, 5.12; $P=.003$; Table). When compared among the 3 categories of patients with AH (clinically suspected, biopsy-proved lymphocytic, and biopsy-proved granulomatous), the volume was not different between the lymphocytic (mean, $5.08 \mathrm{~cm}^{3}$ ) and granulomatous (mean, $5.28 \mathrm{~cm}^{3} ; P=.78$ ) forms but was significantly lower in the clinically suspected form (mean, $3.23 \mathrm{~cm}^{3} ; P=.007$ versus the granulomatous form and $P=.105$ versus lymphocytic hypophysitis).

Gadolinium uptake was higher in $\mathrm{AH}$ than in adenomas (odds ratio, $0.59 ; P=.011$; Table). Most patients with $\mathrm{AH}$ (on-line Table) showed avid enhancement of the sellar mass, similar to the postcontrast medium signal-intensity change in the cavernous sinus. Heterogeneity of the gadolinium uptake was found to be associated with adenomas (odds ratio, 4.31; $P=.041$; Table). About half of the patients with adenoma showed a heterogeneous enhancement, compared with only a minority of patients with $\mathrm{AH}$ (on-line Table).

Asymmetric expansion of the sellar lesion strongly favored a diagnosis of adenoma (odds ratio, 12.1; $P=.001$; Table). Asymmetry was present in most patients with adenoma and in only $4 \%$ of patients with AH (on-line Table). 


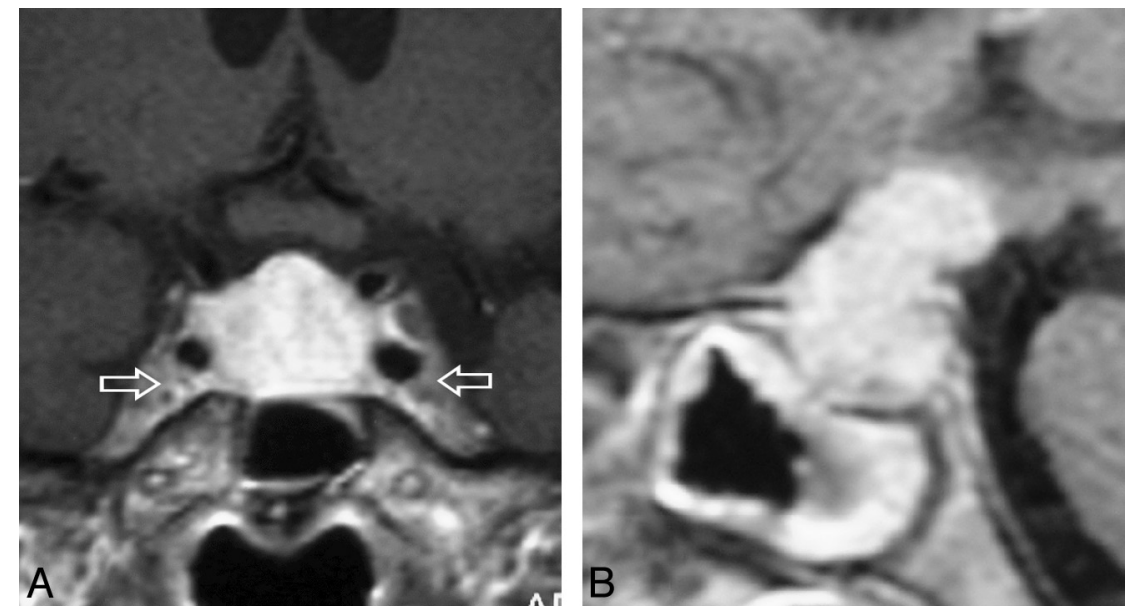

Fig 1. Gadolinium-enhanced coronal $(A)$ and sagittal $(B) T 1$-weighted MR images, sellar sections, in a patient with $A H$. $A$, A perfectly symmetrically enlarged and homogeneously enhancing gland. Note that the enhancement is stronger than that of the adjacent cavernous sinuses (arrows). $B$, Grossly enlarged and avidly enhancing pituitary stalk that exerts a mass effect on the hypothalamus. Note the generalized mucosal thickening in the sphenoid sinus.
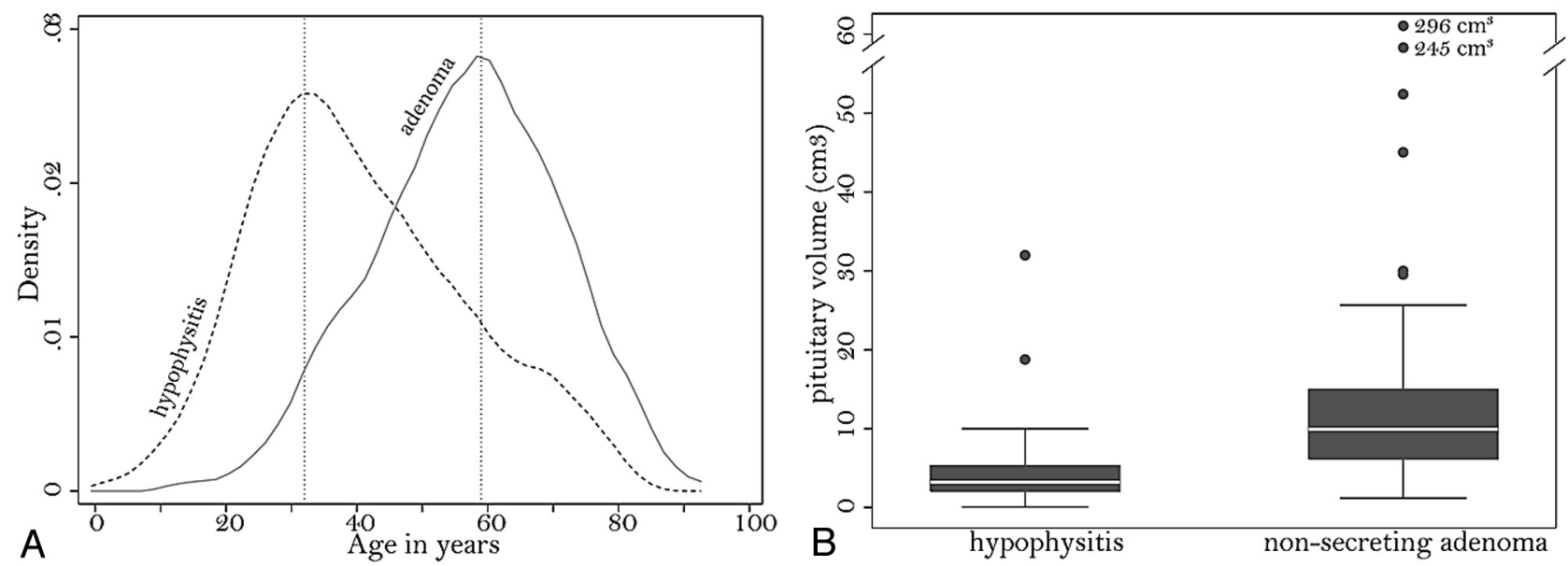

Fig 2. A, Kernel attenuation estimate of the age distribution in patients with AH (dashed line) or nonsecreting pituitary adenoma (continuous line). B, Boxplots show the distribution of pituitary volume in patients with hypophysitis or adenoma. Each box represents the middle half of the distribution (interquartile range) and contains a solid line to indicate the median. The top (and bottom) whisker line extends from the 75th (and 25th) percentile to a calculated value corresponding to 1.5 times the interquartile range. The points outside the ends of the whiskers indicate outliers.

Loss of the normal posterior pituitary bright signal intensity favored a diagnosis of $\mathrm{AH}$ (odds ratio, $0.09 ; P=.007$; Table). In contrast, the posterior pituitary bright spot was conserved in $97 \%$ of patients with adenoma (on-line Table).

A thickened pituitary stalk was highly indicative of $\mathrm{AH}$ (odds ratio, $0.005 ; P<.001$; Table), yielding the largest regression coefficient $(\beta,-5.31)$. The stalk was enlarged in most patients with $\mathrm{AH}$ (ranging from 4 to $11 \mathrm{~mm}$ ) but in only $1 \%$ of adenomas (on-line Table). Last, the presence of mucosal swelling in the sphenoid sinus supported a diagnosis of adenoma (odds ratio, $8.61 ; P=.028$; Table).

Assigning a signed number to each covariate, proportional to its regression coefficient, yielded a cumulative score for each patient that summarized the predictive diagnostic ability of the model. The possible values of the score ranged from a minimum of -13 to a maximum of +8 . In $\mathrm{AH}$ (Fig $3 A$, dotted line), the score ranged from -13 to +2 , had a median of -5 , and comprised most of the patients $(75 \%)$ with values smaller than -2 . In nonsecreting adenomas (Fig $3 A$, solid line), the score ranged from -2 to +8 , had a median of +4 , and comprised most of the patients $(75 \%)$ with values greater than +2 .

The cumulative score had a global performance (ie, AUC) of 0.9917 (Fig 3B). When 1 was chosen as the classification cut- point the score correctly classified $97 \%$ of the patients, with a sensitivity of $92 \%$, a specificity of $99 \%$, a positive predictive value of $97 \%$, and a negative predictive value of $97 \%$ (Fig 3B).

The score in adenoma (median, 4; mean, 3.77) was significantly higher than that in patients with hypophysitis, either when compared with the $3 \mathrm{AH}$ categories combined (median, -5 ; mean, $-4.9 ; P<.00001$; Fig $3 C$ ) or when compared separately with clinically suspected AH (median, -6; mean, $-5.81 ; P<.0001)$, biopsy-proved lymphocytic hypophysitis (median, -5 ; mean, $-4.67 ; P<.0001$ ), or biopsy-proved granulomatous hypophysitis (median, -4 ; mean, $-3.69 ; P<.0001)$. When compared among the 3 categories of patients with $\mathrm{AH}$, the score was similar in biopsy-proved lymphocytic hypophysitis and biopsyproved granulomatous hypophysitis $(P=.2$, Fig $3 C)$, 

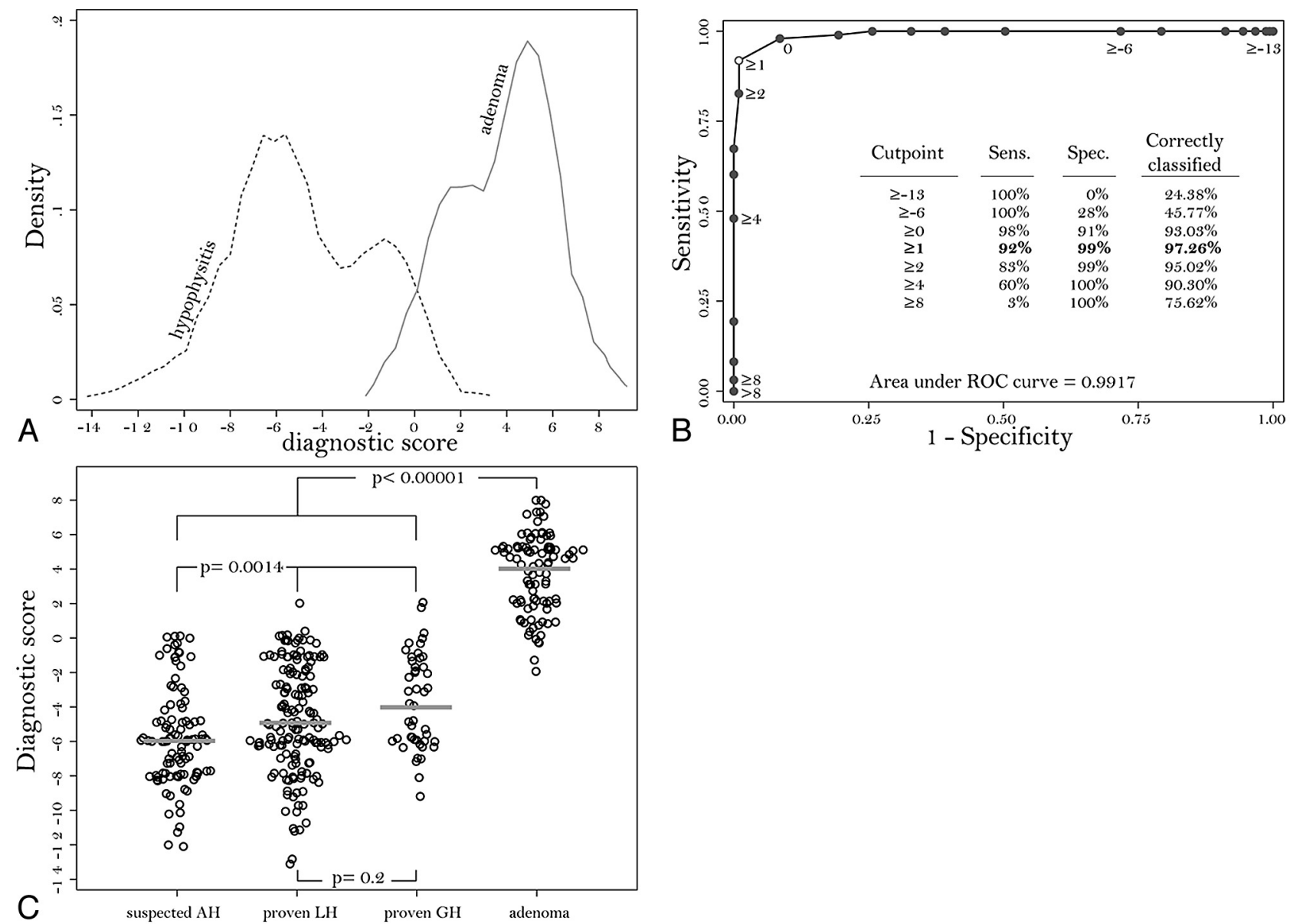

Fig 3. A, Kernel attenuation estimate of the score distribution in patients with $\mathrm{AH}$ (dashed line) or nonsecreting pituitary adenoma (continuous line). B, ROC curve shows sensitivity (Sens.) and 1-specificity (Spec.) obtained by using each value of the score (from -13 to +8 ) as a possible classification cut-point for the diagnosis. The best cut-point is 1 , which classifies correctly $97.26 \%$ of the patients. The AUC, representing the overall performance of the score, is 0.9917 . C, Dotplot shows the distribution of the score in suspected AH, biopsy-proved lymphocytic hypophysitis (LH), biopsy-proved granulomatous hypophysitis $(\mathrm{GH})$, and nonsecreting pituitary adenoma.

whereas it was lower in clinically suspected $\mathrm{AH}$ than in the 2 histologic categories $(P=.0014$, Fig 3C).

\section{Discussion}

This study reports the development of a new score that increases the probability of differentiating $\mathrm{AH}$ from nonsecreting pituitary adenoma before surgery. This distinction is crucial for patient management because AH usually can be treated medically, whereas adenomas most often require surgery, because the score of $\geq 1$ suggests a diagnosis of adenoma, whereas a score of $\leq 0$ suggests a diagnosis of $\mathrm{AH}$. This score classified $>95 \%$ of the patients, thus representing a significant improvement over the current $60 \%$ value. $^{12}$

The score is mainly based on pituitary MR imaging, a technique that has greatly improved the differential diagnosis of sellar lesions due to its exquisite delineation of anatomic details and differences in signal intensity from soft tissues. MR imaging of the sella typically includes precontrast and postgadolinium T1-weighted sequences in both the coronal and sagittal planes. ${ }^{17}$ T2-weighted and fat-suppressed sequences are also useful, specifically in the search of clival bone marrow edema, which has been reported in some cases of hypophysitis, ${ }^{31}$ but these sequences are not routinely performed and thus could not be included in our study.

AH displays MR imaging features that closely reflect the underlying histopathology. Lymphocytic and granulomatous hypophysitis are characterized by lymphoplasmacytic infiltration, destruction of endocrine cells, interstitial widening and fibrosis, hypervascularity, and multinucleated giant cells (the latter prominent in the granulomatous and rare in the lymphocytic form). In keeping with these pathologic changes, the MR imaging features typical of $\mathrm{AH}$ were a symmetric enlargement of the pituitary gland, a homogeneous appearance both on pre- and postgadolinium images, and an intense gadolinium enhancement. In contrast, pituitary adenomas were typically asymmetric as they sprout toward the suprasellar cistern and cavernous sinus ${ }^{32}$; showed a heterogeneous enhancement, likely a reflection of inner cystic or necrotic areas ${ }^{32}$; and had a lower gadolinium uptake than the normal adenohypophysis, consistent with the notion that adenomas have lower vascular attenuations than the normal pituitary tissue. ${ }^{33}$ A strong enhancement is to be expected only in the presence of secondary inflammatory changes, which are rare (approximately $1 \%$ ) in nonfunctioning adenomas. ${ }^{34}$

Consistent with the original report in a small case series, ${ }^{27}$ this study also found that a thickened pituitary stalk is typical for $\mathrm{AH}$ and strongly favored a diagnosis of $\mathrm{AH}$ over that of adenoma. An enlarged pituitary stalk can be found in a variety of diseases, such as germinoma, lymphoma, tuberculosis, sarcoidosis, or Langerhans cell histiocytosis, ${ }^{35}$ but its presence in 
the absence of systemic infections suggests a diagnosis of hypophysitis.

Another MR imaging feature highly indicative of $\mathrm{AH}$ rather than adenoma was the loss of the posterior pituitary bright spot. The normal posterior pituitary gland appears bright on T1-weighted images, likely because of its rich content of vasopressin neurosecretory granules. ${ }^{36}$ This brightness was frequently lost in $\mathrm{AH}$, suggesting a direct autoimmune involvement of the neurohypophysis. The bright spot was conserved in the overwhelming majority of adenomas, and even when displaced by the large tumor size (70\% of patients have bright spot displacement for adenomas with a diameter of $>20$ $\mathrm{mm}^{37}$ ), the bright spot remained visible.

Several additional MR imaging features have been reported in individual cases of $\mathrm{AH}$ but proved not contributory to the final predictive score in this study. The dural tail sign, for example, was originally reported in 4 of 5 patients with $\mathrm{AH}^{21}$ but is also seen in approximately one third of patients with pituitary adenoma. ${ }^{38}$ The sign is now considered nonspecific and indicative of venous congestion rather than meningeal inflammation. ${ }^{39}$ An extension or even infiltration of the pituitary lesion into the basal hypothalamus was originally reported in 5 of 9 patients with $\mathrm{AH}^{27}$ but it has been rarely described in subsequent publications. Inflammatory changes of the cavernous portion ( $\mathrm{C} 4$ segment) of the internal carotid artery have been reported in $\mathrm{AH},{ }^{40}$ but their relevance in the differential diagnosis of sellar masses remains to be clarified.

The pituitary size was larger in adenomas than in AH. This difference likely reflects an ascertainment bias, considering that adenomas present later $\left(23 \pm 35\right.$ months $\left.^{41}\right)$ than $\mathrm{AH}$ $\left(10 \pm 18\right.$ months $\left.^{8}\right)$, where the degree of hypopituitarism can be disproportionate to the size of the pituitary mass. ${ }^{42}$ Most interesting, pituitary volumes were smaller in clinically suspected $\mathrm{AH}$ than in the more advanced surgically treated lymphocytic and granulomatous forms.

The clinical feature that proved most useful in differentiating $\mathrm{AH}$ from adenomas was pregnancy. AH shows, in fact, a striking temporal association with late pregnancy or early postpartum, which at the moment remains unexplained. Pregnancy affects the pituitary gland significantly. The adenohypophysis increases by approximately $30 \%$ over its pregestational volume, peaking at day 3 after delivery ${ }^{43}$ as a consequence of hypertrophy and hyperplasia of lactotroph cells induced by placental estrogens. The neurohypophysis loses its normal bright spot during the third trimester, ${ }^{44}$ though the dimensions of the pituitary stalk and neurohypophysis remain unchanged during normal pregnancy. On the other hand, adenomas are not notably affected by pregnancy. Despite the fact that the lactotroph expansion can lead to visual disturbances and headache in patients with a pre-existing nonsecreting adenoma, pregnancy per se does not increase the adenoma size. ${ }^{45}$ Diabetes insipidus, though not considered in this study, is another useful feature in the differential diagnosis: Its presence strongly suggests that the pituitary mass is not an adenoma. ${ }^{46}$

It is important to discuss some limitations and possible improvements of this study. The quality of MR images for published patients with $\mathrm{AH}$, which were the majority, varied greatly. Although we reviewed all published MR images to confirm and extend the results described in the text of the article, it was clear that printed images are rarely a good substitute for original films. The study exclusively compared AHs to adenomas, which represent the most common entity among the nonsecreting pituitary masses. ${ }^{47}$ There are, however, other lesions of the sella turcica that can mimic AH both clinically and radiologically, such as secondary forms of hypophysitis, ${ }^{48}$ pituitary germinoma, ${ }^{49}$ and lymphoma, ${ }^{50}$ for which this score might be less useful. The instrumentation used to obtain MR images also varied.

The study that first analyzed systematically the MR imaging appearances of $\mathrm{AH}^{20}$ used 1.5T superconducting magnets. More recent studies use higher field-strength systems (3T), which allow thinner sectioning while maintaining high signalintensity-to-noise ratios. Future studies may thus consider more accurate volumetric measurements, more subtle signalintensity changes, and smaller focal pathology. However and most important, in our experience, dedicated pituitary MR imaging with thin-section $(\leq 3 \mathrm{~mm}$ ) multiplanar (coronal and sagittal) pre- and postcontrast images and the use of fatsuppressed sequences are by far not yet universally applied, a finding that constitutes the most severe limitation of diagnostic imaging of pituitary pathologies today. Finally, the score we developed was not validated by using data from other institutions, given the rarity of $\mathrm{AH}$, but will be tested and refined as the recognition of $\mathrm{AH}$ broadens. For example, the score could be expanded by the inclusion of pituitary antibody measurements ${ }^{11}$ or other MR imaging features, such as the dynamic enhancement characteristics, ${ }^{51}$ as they become available.

\section{Conclusions}

We report a convenient clinicoradiologic scoring system to differentiate $\mathrm{AH}$ from pituitary adenoma before any surgical intervention. This score could serve as a general tool in the evaluation of pituitary masses and improve the management of patients with $\mathrm{AH}$ by avoiding unnecessary surgical treatments.

\section{Acknowledgment}

We thank Gloria Villa, data base administrator of the Johns Hopkins Hospital Department of Radiology, for her help in obtaining the MR images.

\section{References}

1. Daly AF, Rixhon M, Adam C, et al. High prevalence of pituitary adenomas: a cross-sectional study in the province of Liege, Belgium. J Clin Endocrinol Metab 2006;91:4769-75. Epub 2006 Sep 12

2. Ezzat S, Asa SL, Couldwell WT, et al. The prevalence of pituitary adenomas: a systematic review. Cancer 2004;101:613-19

3. Biller BM, Swearingen B, Zervas NT, et al. A decade of the Massachusetts General Hospital Neuroendocrine Clinical Center. J Clin Endocrinol Metab 1997;82:1668-74

4. Chanson P, Brochier S. Non-functioning pituitary adenomas. J Endocrinol Invest 2005;28:93-99

5. Asa SL. Practical pituitary pathology: what does the pathologist need to know? Arch Pathol Lab Med 2008;132:1231-40

6. Ferrante E, Ferraroni M, Castrignano T, et al. Non-functioning pituitary adenoma database: a useful resource to improve the clinical management of pituitary tumors. Eur J Endocrinol 2006;155:823-29

7. Dekkers OM, Pereira AM, Roelfsema F, et al. Observation alone after transsphenoidal surgery for nonfunctioning pituitary macroadenoma. J Clin Endocrinol Metab 2006;91:1796-801. Epub 2006 Feb 28

8. Gutenberg A, Buslei R, Fahlbusch R, et al. Immunopathology of primary hypophysitis: implications for pathogenesis. Am J Surg Pathol 2005;29:329-38 
9. Caturegli P, Newschaffer C, Olivi A, et al. Autoimmune hypophysitis. Endocr Rev 2005;26:599-614

10. McKeel DW. Primary hypothyroidism and hypopituitarism in a young woman: pathological discussion. Am J Med 1984;77:326-29

11. Caturegli P. Autoimmune hypophysitis: an underestimated disease in search of its autoantigen(s). J Clin Endocrinol Metab 2007;92:2038-40

12. Leung GK, Lopes MB, Thorner MO, et al. Primary hypophysitis: a singlecenter experience in 16 cases. J Neurosurg 2004;101:262-71

13. Miyake I, Takeuchi Y, Kuramoto T, et al. Autoimmune hypophysitis treated with intravenous glucocorticoid therapy. Intern Med 2006;45:1249-52. Epub 2006 Dec 1

14. Arafah BM, Nasrallah MP. Pituitary tumors: pathophysiology, clinical manifestations and management. Endocr Relat Cancer 2001;8:287-305

15. Minniti G, Jaffrain-Rea ML, Osti M, et al. Radiotherapy for nonfunctioning pituitary adenomas: from conventional to modern stereotactic radiation techniques. Neurosurg Rev 2007;30:167-75

16. Seidenwurm DJ. Neuroendocrine imaging. AJNR Am J Neuroradiol 2008;29:613-15

17. Connor SE, Penney CC. MRI in the differential diagnosis of a sellar mass. Clin Radiol 2003;58:20-31

18. Gutenberg A, Hans V, Puchner MJ, et al. Primary hypophysitis: clinicalpathological correlations. Eur J Endocrinol 2006;155:101-07

19. Lury KM. Inflammatory and infectious processes involving the pituitary gland. Top Magn Reson Imaging 2005;16:301-06

20. Pressman EK, Zeidman SM, Reddy UM, et al. Differentiating lymphocytic adenohypophysitis from pituitary adenoma in the peripartum patient. J Reprod Med 1995;40:251-59

21. Ahmadi J, Meyers GS, Segall HD, et al. Lymphocytic adenohypophysitis: contrast-enhanced MR imaging in five cases. Radiology 1995;195:30-34

22. Perez-Nunez A, Miranda P, Arrese I, et al. Lymphocytic hypophysitis with cystic MRI appearance. Acta Neurochir (Wien) 2005;147:1297-300

23. Saiwai S, Inoue Y, Ishihara T, et al. Lymphocytic adenohypophysitis: skull radiographs and MRI. Neuroradiology 1998;40:114-20

24. Johnsen DE, Woodruff WW, Allen IS, et al. MR imaging of the sellar and juxtasellar regions. Radiographics 1991;11:727-58

25. Kucharczyk W, Davis DO, Kelly WM, et al. Pituitary adenomas: highresolution MR imaging at 1.5 T. Radiology 1986;161:761-65

26. Simmons GE, Suchnicki JE, Rak KM, et al. MR imaging of the pituitary stalk: size, shape, and enhancement pattern. AJR Am J Roentgenol 1992;159:375-77

27. Honegger J, Fahlbusch R, Bornemann A, et al. Lymphocytic and granulomatous hypophysitis: experience with nine cases. Neurosurgery 1997;40:713-22

28. Wallace EW. The dural tail sign. Radiology 2004;233:56-57

29. Royston P. Multiple imputation of missing values. Stata Journal 2004;4:227-41

30. Sun GW, Shook TL, Kay GL. Inappropriate use of bivariable analysis to screen risk factors for use in multivariable analysis. J Clin Epidemiol 1996;49:907-16

31. Kartal I, Yarman S, Tanakol R, et al. Lymphocytic panhypophysitis in a young man with involvement of the cavernous sinus and clivus. Pituitary 2007;10:75-80
32. Bonneville JF, Bonneville F, Cattin F. Magnetic resonance imaging of pituitary adenomas. Eur Radiol 2005;15:543-48

33. Jugenburg M, Kovacs K, Stefaneanu L, et al. Vasculature in nontumorous hypophyses, pituitary adenomas, and carcinomas: a quantitative morphologic study. Endocr Pathol 1995;6:115-24

34. Heshmati HM, Kujas M, Casanova S, et al. Prevalence of lymphocytic infiltrate in $\mathbf{1 4 0 0}$ pituitary adenomas. Endocr J 1998;45:357-61

35. Lipscombe L, Asa SL, Ezzat S. Management of lesions of the pituitary stalk and hypothalamus. The Endocrinologist 2003;13:38-51

36. Lee MH, Choi HY, Sung YA, et al. High signal intensity of the posterior pituitary gland on T1-weighted MR images: correlation with plasma vasopressin concentration to water deprivation. Acta Radiol 2001;42:129-34

37. Bonneville F, Narboux Y, Cattin F, et al. Preoperative location of the pituitary bright spot in patients with pituitary macroadenomas. AJNR Am J Neuroradiol 2002;23:528-32

38. Nakasu Y, Nakasu S, Ito R, et al. Tentorial enhancement on MR images is a sign of cavernous sinus involvement in patients with sellar tumors. AJNR Am J Neuroradiol 2001;22:1528-33

39. Guermazi A, Lafitte F, Miaux Y, et al. The dural tail sign: beyond meningioma. Clin Radiol 2005;60:171-88

40. Melgar MA, Mariwalla N, Gloss DS, et al. Recurrent lymphocytic hypophysitis and bilateral intracavernous carotid artery occlusion: an observation and review of the literature. Neurol Res 2006;28:177-83

41. Drange MR, Fram NR, Herman-Bonert V, et al. Pituitary tumor registry: a novel clinical resource. J Clin Endocrinol Metab 2000;85:168-74

42. Guay AT, Agnello V, Tronic BC, et al. Lymphocytic hypophysitis in a man J Clin Endocrinol Metab 1987;64:631-34

43. Dinc H, Esen F, Demirci A, et al. Pituitary dimensions and volume measurements in pregnancy and post partum: MR assessment. Acta Radiol 1998;39:64-69

44. Corenblum B. Pituitary disease and pregnancy. Available at: http://www. emedicine.com/Med/topic3264.htm. Accessed May 28, 2009

45. Molitch ME. Pituitary disorders during pregnancy. Endocrinol Metab Clin North Am 2006;35:99-116, vi

46. Lamberts SW, de Herder WW, van der Lely AJ. Pituitary insufficiency. Lancet 1998;352:127-34

47. Freda PU, Post KD. Differential diagnosis of sellar masses. Endocrinol Metab Clin North Am 1999;28:81-117, vi

48. Sautner D, Saeger W, Ludecke DK, et al. Hypophysitis in surgical and autoptical specimens. Acta Neuropathol 1995;90:637-44

49. Houdouin L, Polivka M, Henegar C, et al. Pituitary germinoma and lymphocytic hypophysitis: a pitfall-report of two cases[in French]. Ann Pathol 2003;23:349-54

50. Giustina A, Gola M, Doga M, et al. Clinical review 136: primary lymphoma of the pituitary-an emerging clinical entity. J Clin Endocrinol Metab 2001;86:4567-75

51. Sato N, Sze G, Endo K. Hypophysitis: endocrinologic and dynamic MR findings. AJNR Am J Neuroradiol 1998;19:439-44 